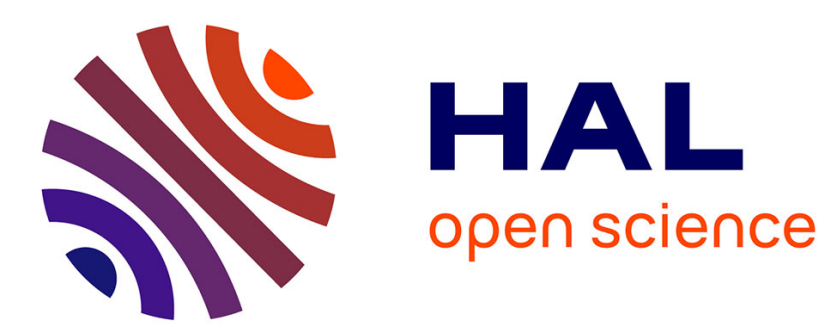

\title{
Stimulated Raman scattering from longitudinal and transverse polariton modes in a damped medium
}

\author{
R. Reinisch, S. Biraud-Laval, N. Paraire
}

\section{To cite this version:}

R. Reinisch, S. Biraud-Laval, N. Paraire. Stimulated Raman scattering from longitudinal and transverse polariton modes in a damped medium. Journal de Physique, 1976, 37 (3), pp.227-232. 10.1051/jphys:01976003703022700 . jpa-00208414

HAL Id: jpa-00208414

https://hal.science/jpa-00208414

Submitted on 1 Jan 1976

HAL is a multi-disciplinary open access archive for the deposit and dissemination of scientific research documents, whether they are published or not. The documents may come from teaching and research institutions in France or abroad, or from public or private research centers.
L'archive ouverte pluridisciplinaire HAL, est destinée au dépôt et à la diffusion de documents scientifiques de niveau recherche, publiés ou non, émanant des établissements d'enseignement et de recherche français ou étrangers, des laboratoires publics ou privés. 


\title{
STIMULATED RAMAN SCATTERING FROM LONGITUDINAL AND TRANSVERSE POLARITON MODES IN A DAMPED MEDIUM
}

\author{
R. REINISCH, S. BIRAUD-LAVAL and N. PARAIRE \\ Institut d'Electronique Fondamentale, Laboratoire associé au C.N.R.S. \\ Université Paris XI, Bât. 220, 91405 Orsay, France
}

(Reçu le 27 mai 1975, révisé le 10 octobre 1975, accepté le 29 octobre 1975)

\begin{abstract}
Résumé. - L'étude théorique de l'excitation de polaritons par effet Raman à l'aide de la méthode à deux faisceaux est effectuée en écrivant les équations de propagation pour les champs associés aux faisceaux laser, Stokes et aux polaritons. Le calcul est fait sans supposer a priori l'onde de polarisation transversale, et en tenant compte de l'amortissement des vibrations du réseau cristallin. Ces équations sont linéarisées par une approximation bien adaptée à la méthode à deux faisceaux, dans laquelle les faisceaux laser et Stokes doivent être traités symétriquement. Nous montrons que l'onde associée aux polaritons résulte de la superposition d'une onde libre, qui est amortie, et d'une onde forcée pour laquelle on ne connaît pas a priori de relation de dispersion. On définit la courbe de dispersion des polaritons excités par effet Raman stimulé à partir des maxima de l'interaction observée dans le cristal. Bien que l'amortissement existe, on observe que la courbe de dispersion des polaritons est toujours très proche de celle obtenue en négligeant l'amortissement.
\end{abstract}

\begin{abstract}
Polaritons excited by Raman effect with the two beam method are studied from a theoretical point of view solving propagation equations for laser, Stokes and polariton fields. The calculation is performed without any assumption on the polarization wave transversality and taking into account lattice vibration damping. The equations are linearized by an approximation well adapted to the two-beam method, in which the laser and Stokes beams must be symmetrically considered. We show that the polariton wave is the superposition of a free wave, which is damped, and a driven one for which no dispersion relation is known a priori. We define the dispersion curve for polaritons excited by stimulated Raman effect from the maxima of the interaction observed in the sample. Although damping is present, the polariton dispersion curve is always found to be very close to that obtained when damping is neglected.
\end{abstract}

1. Introduction. - In a crystal, infrared-active lattice vibrations strongly interact with light waves when their frequencies and wavevectors are nearly equal [1]. The associated mixed particles are known as polaritons. If these optical vibration modes are Raman active too, one can study polaritons by spontaneous or stimulated Raman scattering and get by this way the polariton dispersion curve.

Since polaritons only exist in the vicinity of an absorption band of the crystal, damping of lattice vibrations is an important parameter when studying polaritons. Theoretical studies which take this effect into account have been published to describe several kinds of experimental conditions : for spontaneous Raman scattering, Benson and Mills [2] and, in a more straightforward way, Barker and Loudon [3] show that the polariton dispersion curve is accurately described by the dispersion relation appropriate to the case where damping is ignored; for stimulated Raman effect, with the usual parametric approxima- tion (in which the pump is undepleted), Shen [4] arrives at the same conclusion.

In this paper, we discuss the definition of the dispersion curve for polaritons excited by the two beam method (T.B.M.) which becomes wide-spread to achieve stimulated Raman scattering [5, 6] or more generally non-linear optic experiments [7-9]. In this method, polaritons (or any other kind of elementary excitations) are created by sending two light beams into the crystal; the difference between their frequencies is just equal to that of the expected polaritons. A quantum calculation [10] shows that the excited polaritons are in a Glauber state, i.e. coherent from the very beginning of the interaction. This justifies the use of Maxwell's equations. But in this case, the usual theory for stimulated Raman effect [4] is no longer valid as the two incident beams must be considered in a symmetrical way.

In order to define the polariton dispersion curve, we first calculate the polarization wave electric field 
without assuming it to be purely transverse, which is the usual assumption. The expression thus obtained accounts for longitudinal phonon modes as well as transverse polariton modes (crystal anisotropy is not included here but the same method can be generalized to describe oblique polaritons [11]). The theoretical results are discussed with respect to the specific configuration corresponding to experiments we have performed in quartz [12].

This study illustrates the difference between the dispersion curves obtained from I.R. absorption and those plotted from Raman experiments.

2. Polariton field and dispersion curves. - In order to describe T.B.M., we consider a cubic non centrosymmetric crystal and two coherent light beams, the electric field of which are $\boldsymbol{E}_{1}$ and $\boldsymbol{\delta}_{2}$, with frequencies $\omega_{1}$ and $\omega_{2} \quad\left(\omega_{1}>\omega_{2}\right)$ and wavevectors $\mathbf{k}_{1}$ and $\mathbf{k}_{2}$. Assuming that the medium is perfectly transparent for the frequencies $\omega_{1}$ and $\omega_{2}, k_{1}$ and $k_{2}$ are real quantities. These two light beams generate inside the crystal a polarization wave at frequency $\omega_{3}=\omega_{1}-\omega_{2}$, which can be described by vibrationnal coordinates $q_{h}^{m}$ (the optical modes are enumerated by the superscript $m$ ) and by the electric field cartesian components $\varepsilon_{3 h}$, obeying respectively the following coupled equations [13] :

$$
\begin{aligned}
\frac{\partial^{2} q_{h}^{m}}{\partial t^{2}}+\Gamma_{m} \frac{\partial q_{h}^{m}}{\partial t}+ & \omega_{m}^{2} q_{h}^{m}=\frac{e_{m}}{M_{m}} \varepsilon_{3 h}+ \\
& +\frac{V}{N M_{m}} \alpha_{h i j}^{m}\left(\omega_{3}=\omega_{1}-\omega_{2}\right) \varepsilon_{1 i} \varepsilon_{2 j}^{*}
\end{aligned}
$$$$
\Delta \varepsilon_{3 h}-\left(\operatorname{grad} . \operatorname{div} \boldsymbol{E}_{3}\right)_{h}-\frac{\varepsilon_{\infty}}{c^{2}} \frac{\partial^{2} \varepsilon_{3 h}}{\partial t^{2}}=\frac{1}{\varepsilon_{0} c^{2}} \frac{\partial^{2}}{\partial t^{2}} \times
$$$$
\times\left(\sum_{m} \frac{N e_{m}}{V} q_{h}^{m}+\xi_{h i j}\left(\omega_{3}=\omega_{1}-\omega_{2}\right) \varepsilon_{1 i} \varepsilon_{2 j}^{*}\right)
$$

where we use the convention that repeated coordinate indices must be summed. $\Gamma_{m}, e_{m}$ and $M_{m}$ are respectively the damping constant, effective charge and reduced mass relative to the $m$ th optical mode; $N / V$ is the concentration of unit cells in the crystal; $\varepsilon_{0}$ is the vacuum dielectric constant, $\varepsilon_{\infty}$ the crystal high frequency permittivity.

$\alpha_{h i j}^{m}$ and $\xi_{h i j}$ are polarisability derivatives :

$$
\alpha_{h i j}^{m}=\left(\partial \alpha_{i j} / \partial q_{h}^{m}\right)_{q_{h}^{m}=0} \quad \xi_{h i j}=\left(\partial \alpha_{i j} / \partial \varepsilon_{3 h}\right)_{\varepsilon_{3 h}=0} .
$$

Let us assume that the fields $\boldsymbol{\varepsilon}_{1}$ and $\boldsymbol{\varepsilon}_{2}$ can be expressed in the following form $[14,15]$ :

$$
\boldsymbol{\delta}_{v}=\mathbf{e}_{v} E_{v}(\mathbf{r}) \mathrm{e}^{j\left(\omega_{v} t-\mathbf{k}_{v} \cdot \mathbf{r}\right)} \quad(v=1,2)
$$

where $\mathbf{e}_{v}$ is a unit vector. This implies that the fields $\boldsymbol{\varepsilon}_{v}(v=1,2)$ keep a fixed (transverse) polarization as they propagate in the nonlinear medium.

As the interaction occurs, the higher frequency beam $\left(\boldsymbol{\varepsilon}_{1}\right)$ is depleted while the lower frequency one $\left(\boldsymbol{\varepsilon}_{2}\right)$ is amplified. Under these conditions, the product $E_{1} E_{2}^{*}$ can be assumed constant [12] and this approximation linearizes eq. (1).

Let $q_{h}^{m}$ and $\varepsilon_{3 h}$ have a space and time dependence :

$$
\begin{aligned}
& q_{h}^{m}=Q_{h}^{m}(\mathbf{r}) \mathrm{e}^{j \omega_{3} t} \\
& \varepsilon_{3 h}=E_{3 h}(\mathbf{r}) \mathrm{e}^{j \omega_{3} t} .
\end{aligned}
$$

We get for $E_{3 h}$ the following equation :

$$
\begin{aligned}
\Delta E_{3 h} & -\left(\operatorname{grad} . \operatorname{div} \mathbf{E}_{3}\right)_{h}+k^{2} E_{3 h}= \\
& =-\frac{\omega_{3}^{2}}{c^{2}} 2 \chi_{h i j}\left(\omega_{3}=\omega_{1}-\omega_{2}\right) E_{1} E_{2}^{*} e_{1 i} e_{2 j} \mathrm{e}^{-j \mathbf{k}_{3} \cdot \mathbf{r}}
\end{aligned}
$$

where

$$
2 \varepsilon_{0} \chi_{h i j}\left(\omega_{3}\right)=\xi_{h i j}\left(\omega_{3}\right)+\sum_{m} \frac{\alpha_{h i j}^{m}\left(\omega_{3}\right) e_{m} / M_{m}}{\omega_{m}^{2}-\omega_{3}^{2}+j \omega_{3} \Gamma_{m}}
$$

$k^{2}$ is given by :

$$
\frac{k^{2} c^{2}}{\omega_{3}^{2}}=\varepsilon_{\infty}+\sum_{m} \frac{S_{m} \omega_{m}^{2}}{\omega_{m}^{2}-\omega_{3}^{2}+j \omega_{3} \Gamma_{m}}
$$

with

$$
S_{m} \omega_{m}^{2}=\frac{N e_{m}^{2}}{\varepsilon_{0} N_{m} V}
$$

$\mathbf{k}_{\mathbf{3}}$ which is just equal to $\mathbf{k}_{\mathbf{1}}-\mathbf{k}_{\mathbf{2}}$ is a real quantity.

The wave eq. (3) is solved by assuming that as the two incident beams propagate in the $x \mathrm{O} y$ plane there is no dependence on the $z$ coordinate. Thus, we have two coupled equations for $E_{3 x}(\mathbf{r})$ and $E_{3 y}(\mathbf{r})$, but the equation for $E_{3 z}(\mathbf{r})$ can be solved independently :

$$
\begin{aligned}
\frac{\partial^{2} E_{3 x}}{\partial y^{2}}-\frac{\partial^{2} E_{3 y}}{\partial x \partial y} & +k^{2} E_{3 x}= \\
= & -\frac{\omega_{3}^{2}}{c^{2}} 2 \chi_{x i j} E_{1} E_{2}^{*} e_{1 i} e_{2 j} \mathrm{e}^{-j \mathbf{k}_{3} \cdot \mathbf{r}} \\
\frac{\partial^{2} E_{3 y}}{\partial x^{2}}-\frac{\partial^{2} E_{3 x}}{\partial x \partial y} & +k^{2} E_{3 y}= \\
= & -\frac{\omega_{3}^{2}}{c^{2}} 2 \chi_{y i j} E_{1} E_{2}^{*} e_{1 i} e_{2 j} \mathrm{e}^{-j \mathbf{k}_{3} \cdot \mathbf{r}}
\end{aligned}
$$

$$
\begin{aligned}
\frac{\partial^{2} E_{3 z}}{\partial x^{2}}+\frac{\partial^{2} E_{3 z}}{\partial y^{2}} & +k^{2} E_{3 z}= \\
& =-\frac{\omega_{3}^{2}}{c^{2}} 2 \chi_{z i j} E_{1} E_{2}^{*} e_{1 i} e_{2 j} \mathrm{e}^{-j \mathbf{k}_{3} \cdot \mathbf{r}}
\end{aligned}
$$

From these equations, we obtain for the $\omega_{3}$ field components solutions of the form :

$$
E_{3 h}=a_{h} \mathrm{e}^{-j\left(k_{x} x+k_{y} y\right)}+b_{h} \mathrm{e}^{-j\left(k_{3 x} x+k_{3 y} y\right)}
$$

with

$$
k_{x}^{2}+k_{y}^{2}=k^{2}
$$


$b_{h}$ is known from a particular solution of eq. (6)-(8) :

$$
b_{h}=\frac{2 \omega_{3}^{2}}{c^{2}} \frac{\left(k^{2}-k_{3 h}^{2}\right) \chi_{h i j}-k_{3 h} k_{3 l} \chi_{l i j}}{k^{2}\left(k_{3}^{2}-k^{2}\right)} E_{1} E_{2}^{*} e_{1 i} e_{2 j}
$$

with $l \neq h$ and reminding that $k_{3 z}=0$.

Boundary conditions in the plane $y=0$ lead to the following expression for $a_{h}$ :

$$
\begin{aligned}
& a_{x}=\frac{k_{y}\left[\left(K_{y} k_{3 y}-K^{2}\right) b_{x}-K_{y} k_{3 x} b_{y}\right]}{K^{2} k_{y}-k^{2} K_{y}} \\
& a_{y}=\frac{-k_{x}\left[\left(K_{y} k_{3 y}-K^{2}\right) b_{x}-K_{y} k_{3 x} b_{y}\right]}{K^{2} k_{y}-k^{2} K_{y}} \\
& a_{z}=\frac{\left(k_{3 z}-K_{y}\right) b_{z}}{K_{y}-k_{y}}
\end{aligned}
$$

where $\mathbf{K}$ is the wavevector of a free wave radiated outside the crystal at the polariton frequency $\omega_{3}$. These boundary conditions also imply that

$$
k_{x}=K_{x}=k_{3 x} \text {. }
$$

Then $k_{x}$ and $K_{x}$ are real quantities.

The polariton field given by (9) includes two terms :

i) the first one is proportional to $\left.\mathrm{e}^{-j\left(k_{x} x+k_{y} y\right.}\right)$ in which $k_{y}$ is complex. It represents a free damped wave, with equal amplitude planes parallel to the crystal boundary. This wave is similar to the free wave propagating in an absorbing medium illuminated by an electromagnetic radiation at frequency $\omega_{3}$, since its wavevector $k$ is also given by eq. (5).

ii) the second term is proportional to $\mathrm{e}^{-j\left(k_{3 x} x+k_{3 y} y\right)}$ with $\mathbf{k}_{3}=\mathbf{k}_{1}-\mathbf{k}_{2}$, and describes a wave which is driven by the interaction between the two incident beams inside the crystal. This wave keeps a constant amplitude while it propagates through the medium, and is the only one that can be observed in a T.B.M. experiment, in which the crystal thickness is usually very large with respect to the free wave damping length.

It is worth noting that for the driven wave no dispersion relation $\omega_{3}\left(k_{3}\right)$ has appeared in the calculation, and that the relation given by eq. (5), which is well known in I.R. dispersion theory, is only valid for the free wave. Nevertheless, some authors still admit that this relation describes Raman-excited polariton dispersion, implying that either $k$ or $\omega_{3}$ (or both) is a complex quantity. But considering that $k$ is complex [16-20] and that polaritons propagate with a wavevector $k^{\prime}=\operatorname{Re}(k)$ leads to a discrepancy, as the dispersion curve $\omega_{3}\left(k^{\prime}\right)$ presents turnarounds inconsistent with the experimental results obtained from Raman scattering. This is shown on figure 1 where the curve $\omega_{3}\left(k^{\prime}\right)$ is drawn in solid line. Several experimental points clearly appears beyond the turnarounds, and in particular all the right-angle scattering results.

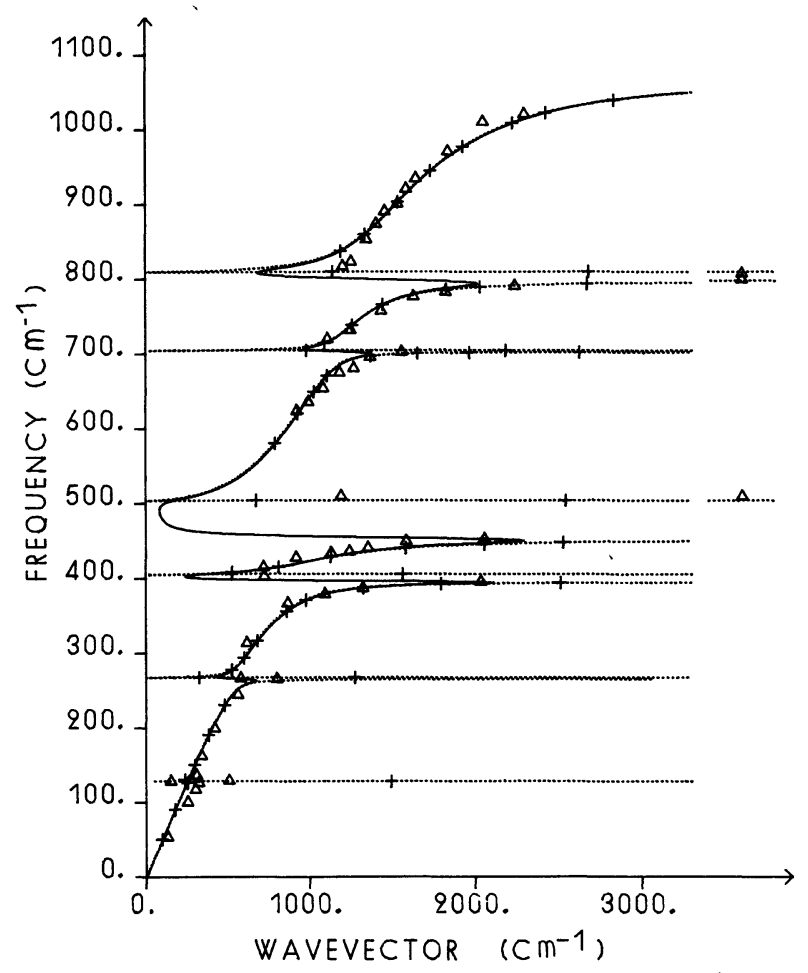

FIG. 1. - Polariton dispersion curve in quartz : Solid line : I.R. dispersion curve $\omega_{3}\left(k^{\prime}\right)$. Triangles : experimental points. Dashed line : curve given by the undamped dispersion relation (18). Crosses : $\Delta W_{2}$ peak position.

In order to avoid this difficulty, some other authors [21-24] assume that $k$ is real and $\omega_{3}$ complex. The curve obtained by plotting $\operatorname{Re}\left(\omega_{3}\right)$ versus $k$ produces no discrepancy with the experimental results, but the application of relation (5) to the case of polaritons excited by Raman effect is no better justified.

As we have already mentionned, the only wave to be observed in a Raman T.B.M. experiment is the driven one, for which no dispersion relation is known. But it is obvious that the intensity of this wave varies with the relative values of $k_{3}$ and $\omega_{3}$, and presents peaks for specific values $K_{3}, \Omega_{3}$ of $k_{3}$ and $\omega_{3}$. It is then consistent to define the polariton dispersion curve from the maxima of $\left|b_{h}\right|^{2}$, by plotting $\Omega_{3}$ versus $K_{3}$.

The main features about polariton dispersion are best appreciated by considering a diatomic crystal. Substituting eq. (5) into $\left|b_{h}\right|^{2}$, the denominator $\left|k^{2}\right|^{2}\left|k_{3}^{2}-k^{2}\right|^{2}$ becomes proportionnal to :

$$
\begin{aligned}
& \left\{\left[\varepsilon_{\infty}\left(\omega_{0}^{2}-\omega_{3}^{2}\right)+S \omega_{0}^{2}\right]^{2}+\varepsilon_{\infty}^{2} \omega_{3}^{2} \Gamma^{2}\right\} \times \\
& \times\left\{\left[\left(\frac{k_{3}^{2} c^{2}}{\omega_{3}^{2}}-\varepsilon_{\infty}\right)\left(\omega_{0}^{2}-\omega_{3}^{2}\right)-S \omega_{0}^{2}\right]^{2}\right. \\
& \left.+\omega_{3}^{2} \Gamma^{2}\left(\frac{k_{3}^{2} c^{2}}{\omega_{3}^{2}}-\varepsilon_{\infty}\right)^{2}\right\} .
\end{aligned}
$$

The two terms proportional to $\Gamma^{2}$ increase monotonously with $\omega_{3}$. As long as $\Gamma$ is not too large (i.e. 
excluding overdamped media) the zeros of the denominator are close to the zeros of :

and

$$
\varepsilon_{\infty}\left(\omega_{0}^{2}-\omega_{3}^{2}\right)+S \omega_{0}^{2}
$$

$$
\left(\frac{k_{3}^{2} c^{2}}{\omega_{3}^{2}}-\varepsilon_{\infty}\right)\left(\omega_{0}^{2}-\omega_{3}^{2}\right)-S \omega_{0}^{2} \text {. }
$$

Provided that the other factors in $\left|b_{h}\right|^{2}$ do not vary rapidly with frequency (this is usually the case), the zeros of expression (15) account for peaks of $\left|b_{h}\right|^{2}$ at a frequency $\Omega_{3 L}$ independent of wavevector $K_{3}$ and thus correspond to longitudinal phonon modes. In the same way, zeros of expression (16) occur for the dispersion relation :

$$
\frac{K_{3}^{2} c^{2}}{\Omega_{3}^{2}}=\varepsilon_{\infty}+\frac{S \omega_{0}^{2}}{\omega_{0}^{2}-\Omega_{3}^{2}}
$$

where no damping is present. Thus, the polariton dispersion curve, defined from the driven wave square amplitude peaks, is very well described by the undamped dispersion relation (17).

This is illustrated on figure 2 where we plot in a $\left(\Omega_{3}, K_{3}\right)$ diagram the curve given by the undamped dispersion relation (17) (dashed line) using GaP parameters [25], the crosses corresponding to the peaks of $\left|b_{h}\right|^{2}$. For comparison, we also draw (solid line) the I.R. dispersion curve $\Omega_{3}(\operatorname{Re}(k))$.

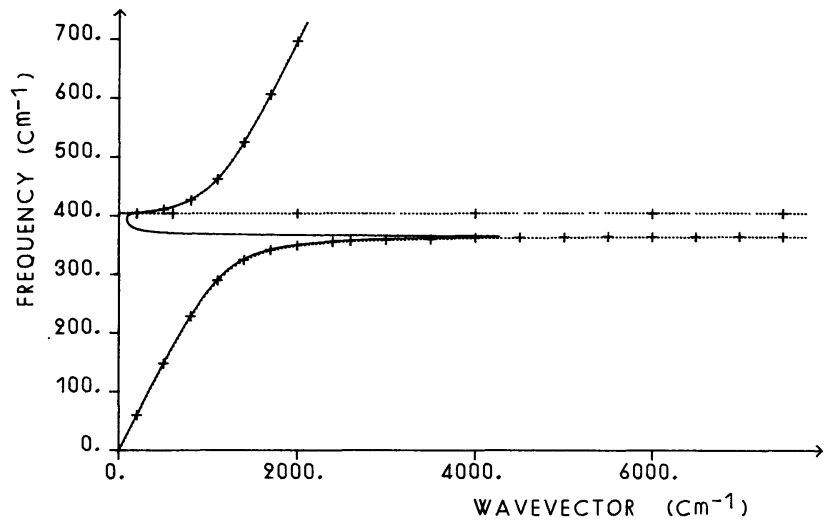

FIG. 2. - Polariton dispersion curve in a diatomic crystal (GaP) : Solid line : I.R. dispersion curve $\omega_{3}\left(k^{\prime}\right)$. Dashed line : curve given by the undamped dispersion relation (17). Crosses : peak position of $\left|b_{h}\right|^{2}$.

These results show that the main frequency dependence in $\left|b_{h}\right|^{2}$ is included in the term $\left|k^{2}\right|^{2}\left|k_{3}^{2}-k^{2}\right|^{2}$ which determines the polariton dispersion curve. This also agrees with the theory given by Barker and Loudon [3] for spontaneous Raman scattering.

Coming back to the case of multiatomic crystals, we can still consider that the dispersion curve defined from the driven wave amplitude peaks is very well described by the undamped relation :

$$
\frac{K_{3}^{2} c^{2}}{\Omega_{3}^{2}}=\varepsilon_{\infty}+\sum_{m} \frac{S_{m} \omega_{m}^{2}}{\omega_{m}^{2}-\Omega_{3}^{2}} .
$$

In order to verify this accurately, one must consider specific configurations. This is done here for quartz which has been experimentally studied by T.B.M. $[5,12]$.

3. Power variations of the incident beams. Application to a quartz crystal. - From an experimental point of view, the direct observation of the driven wave, which needs far infrared detection is much more difficult than measurements performed on the incident beams, at frequencies belonging to the visible range. Under these conditions, in T.B.M., interaction is usually characterized by the relative attenuation of the higher frequency beam or the relative amplification of the lower frequency one. Let us calculate this latter quantity.

The lower-frequency beam $\boldsymbol{\varepsilon}_{2}$ obeys the propagation equation :

$$
\Delta \boldsymbol{\varepsilon}_{2}-\operatorname{grad} \cdot \operatorname{div} \boldsymbol{\varepsilon}_{2}-\frac{n_{2}^{2}}{c^{2}} \frac{\partial^{2} \boldsymbol{E}_{2}}{\partial t^{2}}=\frac{1}{c^{2}} \frac{\partial^{2} \mathscr{T}_{2}^{\mathrm{NL}}}{\partial t^{2}}
$$

$n_{2}$ is the refractive index and $\mathfrak{T}_{2}^{\mathrm{NL}}$ the non linear polarisation at frequency $\omega_{2}$. Components of $\mathfrak{J}_{2}^{\mathrm{NL}}$ are :

$$
\mathfrak{T}_{2 j}^{\mathrm{NL}}=2 \chi_{j i h}^{*}\left(\omega_{2}\right) \varepsilon_{1 i} \varepsilon_{3 h}^{*}+\chi_{j i k l}^{\mathrm{R}} \varepsilon_{1 i} \varepsilon_{1 k}^{*} \varepsilon_{2 l}
$$

where

$$
\chi_{j i k l}^{\mathrm{R}}=\sum_{m} \frac{\alpha_{j i h}^{m}\left(\omega_{2}\right) \alpha_{h k l}^{m}\left(\omega_{3}\right) V / M_{m} N \varepsilon_{0}}{\omega_{m}^{2}-\omega_{3}^{2}+j \omega_{3} \Gamma_{m}} .
$$

Substituting (2) into (19) and assuming that the field amplitude is slowly varying over a wavelength yield the equation :

$$
2 j \mathbf{k}_{2} \cdot \operatorname{grad} E_{2}(\mathbf{r})=\frac{\omega_{2}^{2}}{c^{2}} \mathbf{e}_{2} \cdot \mathbf{P}_{2}^{\mathrm{NL}}
$$

with

and

$$
\begin{gathered}
k_{2}=\frac{n_{2} \omega_{2}}{c} \\
\mathfrak{P}_{2}^{\mathrm{NL}}=\mathbf{P}_{2}^{\mathrm{NL}} \mathrm{e}^{j\left(\omega_{2} t-\mathbf{k}_{2} \mathbf{r}\right)} .
\end{gathered}
$$

As we have shown for the polariton field, the equal amplitude plartes for $\varepsilon_{2}$ can be considered parallel to the crystal boundary, i.e. $E_{2}$ depends only on $y$. Eq. (21) becomes :

$$
\frac{\mathrm{d} E_{2}}{\mathrm{~d} y}=\frac{1}{2 j k_{2 y}} \frac{\omega_{2}^{2}}{c_{2}} \mathrm{e}_{2 j} P_{2 j}^{\mathrm{NL}} .
$$

Substituting (9) and (20) into (22), we get

$$
\frac{\mathrm{d} E_{2}}{\mathrm{~d} y}=\frac{1}{2 j k_{2 y}} \frac{\omega_{2}^{2}}{c^{2}} u_{j i k l}^{*}(y) e_{1 i} e_{1 k} e_{2 l} e_{2 j}\left|E_{1}\right|^{2} E_{2}
$$

with

$$
u_{j i k l}(y)=2 \chi_{j i h}\left(A_{h k l} \mathrm{e}^{-j\left(k_{y}-k_{3 y}\right) y}+B_{h k l}\right)+\chi_{j i k l}^{\mathrm{R}} .
$$


We have introduced here the notations $A_{h k l}$ and $B_{h k l}$ according to the relations :

$$
\begin{aligned}
& a_{h}=A_{h k l} E_{1} E_{2}^{*} e_{1 k} e_{2 l} \\
& b_{h}=B_{h k l} E_{1} E_{2}^{*} e_{1 k} e_{2 l} .
\end{aligned}
$$

The intensity variations are given by the following equation :

$$
\begin{aligned}
\frac{\mathrm{d}\left|E_{2}\right|^{2}}{\mathrm{~d} y}=-\frac{\omega_{2}^{2}}{c^{2} k_{2 y}} \mid & \left.E_{1} E_{2}^{*}\right|^{2} \times \\
& \times \operatorname{Im}\left(u_{j i k l}^{*}(y) e_{1 i} e_{1 k} e_{2 l} e_{2 j}\right) .
\end{aligned}
$$

For a crystal thickness $L$, the net power variation is :

$$
\begin{aligned}
& \Delta W_{2}=\frac{2 \omega_{2} W_{1} W_{2}}{\varepsilon_{0} c^{2} n_{1} n_{2}} \times \\
& \times\left\{\operatorname{Re}\left[2 \chi_{j i h}\left(\omega_{2}\right) A_{h k l} \frac{1-\mathrm{e}^{-j\left(k_{y}-k_{3 y}\right) L}}{k_{y}-k_{3 y}}\right]-\right. \\
& \left.-\operatorname{Im}\left[\left(2 \chi_{j i h}\left(\omega_{2}\right) B_{h k l}+\chi_{j i k l}^{\mathrm{R}}\right) L\right]\right\} e_{1 i} e_{1 k} e_{2 l} e_{2 j} .
\end{aligned}
$$

In eq. (25), the term $\operatorname{Re}(.$.$) comes from the contri-$ bution of the free wave to the power variation $\Delta W_{2}$. As this wave is rapidly damped, this term is only important for a very small crystal thickness. In most cases, crystals used in this kind of experiments are much thicker than the damping length and this contribution is negligible compared with that of the driven wave; the following simplified expression can then be used :

$$
\begin{aligned}
& \Delta W_{2}=-\frac{2 \omega_{2} W_{1} W_{2}}{\varepsilon_{0} c^{2} n_{1} n_{2}} \times \\
& \times \operatorname{Im}\left[\left(2 \chi_{j i h}\left(\omega_{2}\right) B_{h k l}+\chi_{j i k l}^{\mathrm{R}}\right) L\right] e_{1 i} e_{1 k} e_{2 l} e_{2 j} .
\end{aligned}
$$

In the same way, the higher frequency beam depletion $\Delta W_{1}$ can be calculated, showing that Manley Rowe relations :

$$
\frac{\Delta W_{1}}{\omega_{1}}=-\frac{\Delta W_{2}}{\omega_{2}}
$$

are verified.

In eq. (26), the factor $B_{h k l}$ appears again leading for $\Delta W_{2}$ to a denominator proportional to $\left|k^{2}\right|^{2}\left|k_{3}^{2}-k^{2}\right|^{2}$ which mainly determines the peak position, according to the preceding section. As in the case of $\left|b_{h}\right|^{2}$ the other factors in $\Delta W_{2}$ do not vary rapidly with frequency. Peaks of $\Delta W_{2}$ then coincide with those of the driven wave amplitude, and we can define the polariton dispersion curve from the peaks of $\Delta W_{2}$ - this definition is closer to the experimental method - as well as from those of $\left|b_{h}\right|^{2}$.

In order to plot the theoretical dispersion curve defined from the peaks of $\Delta W_{2}$, and to compare it with that given by relation (18), let us consider the case of quartz crystal with the geometry used in the experiments we performed [5, 12]. Figure 3 shows the scattering diagram : beams propagate in the $x \mathrm{O} y$ plane (perpendicular to the optical axis) which is an isotropic plane. The $\omega_{1}$ frequency beam propagates along $\mathrm{O} y$ and is polarized along $\mathrm{O} x$; the $\omega_{2}$ frequency beam propagates at a given angle $\theta$ from $\mathrm{O} y$, allowing non collinear phase-matching and its polarization lies along $\mathrm{O} z$. Under these conditions, as quartz belongs to the $D_{3}$ class, crystal symmetry considerations give as non-zero terms of the $[\alpha]$ and $[\xi]$ tensors in eq. (1) $\alpha_{y x z}$ and $\xi_{y x z}$, and imply $\xi_{y x z}=-\xi_{x y z}$. Furthermore, the elements of the tensor [ $\xi$ ] being frequency independent, obey Kleinman relations,

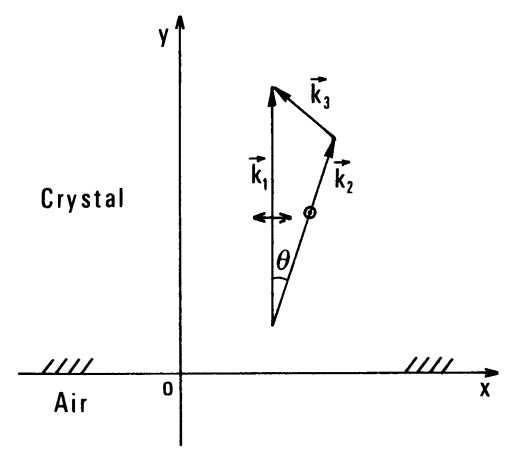

FIG. 3. - Scattering diagram.

so we get $\xi_{y x z}=0$, which agrees with second harmonic generation experiments performed in quartz [26]. On the other hand, some results given by Shen and Bloembergen [27] may be generalized [28] and lead to :

$$
\begin{aligned}
\alpha_{x z y}^{m}\left(\omega_{1}=\omega_{2}+\omega_{3}\right) & =\alpha_{z x y}^{m}\left(\omega_{2}=\omega_{1}-\omega_{3}\right) \\
& =\alpha_{y x z}^{m}\left(\omega_{3}=\omega_{1}-\omega_{2}\right) .
\end{aligned}
$$

In this case, we only observe purely transverse polariton and longitudinal phonon modes.

For this specific configuration, the amplification of the lower frequency beam is therefore given by :

$$
\begin{aligned}
\Delta W_{2}= & \frac{2 \omega_{2} W_{1} W_{2}}{\varepsilon_{0} c^{2} n_{1 x} n_{2 z}} \times \\
& \times \operatorname{Im}\left[\left(4 \chi_{z x y}^{2} \frac{\omega_{3}^{2}}{c^{2}} \frac{k^{2}-k_{3 y}^{2}}{k^{2}\left(k^{2}-k_{3}^{2}\right)}-\chi_{z x x z}^{\mathrm{R}}\right) L\right] .
\end{aligned}
$$

The peak position of $\Delta W_{2}$ in a $\left(\Omega_{3}, K_{3}\right)$ diagram is computed using numerical data from ref. [29] : results are plotted on figure 1 (crosses) where we have also reported the curve given by the dispersion relation (18).

It can be noticed that, even for a multimode crystal such as $\alpha$-quartz, the theoretical $\Delta W_{2}$ maxima are on the curve deduced from (18) and so, the undamped dispersion relation appears to be a very good approximation to describe the polariton dispersion curve obtained by Raman effect.

4. Conclusion. - This theoretical description of the excitation of polaritons by T.B.M. outlines the difference between the dispersion curves observed by I.R. absorption and by Raman scattering. 
When an I.R. wave is incident on an absorbing crystal, a free damped wave propagates inside the medium and the resolution of Maxwell's equations leads to the condition (5) between its frequency and wavevector. This is very different from the case of polaritons excited by the simultaneous presence of photons (beam $\omega_{1}$ ) and photons (beam $\omega_{2}$ ) inside the active medium, for in this case the interaction gives rise to a driven wave, for which no analytical dispersion relation is known a priori. This leads us to define a dispersion relation $K_{3}\left(\Omega_{3}\right)$ as that for which the interaction presents a maximum intensity. A phase matching condition can then be written as a wavevector equality between the driven wavevector $\mathbf{k}_{1}-\mathbf{k}_{2}$ and the wavevector $\mathbf{K}_{3}$.

Acknowledgments. - We would like to thank Professor R. Loudon for helpful discussions, and we acknowledge the continuous interest offered to this work by Dr. G. Chartier.

\section{References}

[1] Born, M. and HuAng, K., Dynamical Theory of Crystal Lattices (Oxford) 1966.

[2] Benson, H. J. and Mills, D. L., Phys. Rev. B 1 (1970) 1678.

[3] BARKer Jr., A. S. and Loudon, R., Rev. Mod. Phys. 44 (1972) 18.

[4] Shen, Y. R., Phys. Rev. A 138 (1965) 1741.

[5] Biraud-Laval, S., J. Physique 35 (1974) 513.

[6] Coffinet, J. P., De Martini, F., Phys. Rev. Lett. 22 (1968) 60.

[7] Lax, B., Aggarwal, R. L., Favrot, G., Appl. Phys. Lett. 23 (1973) 679.

[8] Yang, K. H., Morris, J. R., Richards, P. L., Shen, Y. R., Appl. Phys. Lett. 23 (1973) 669.

[9] Hanna, D. C., Smith, R. C., Stanley, C. R., Opt. Commun. 4 (1971) 300.

[10] Reinisch, R., Biraud-Laval, S., Chartier, G., Paraire, N., Phys. Rev. B 9 (1974) 1861.

[11] Paraire, N., Koster, A., Biraud-Laval, S., Reinisch, R., Phys. Stat. Sol. 68B (1975) 543.

[12] Biraud-Laval, S., Thèse d'Etat, Orsay (1973).

[13] Loudon, R., Light Scattering in Solids (Springer-Verlag, New-York) 1969.

[14] Bloembergen, N., Nonlinear Optics (Benjamin, N. Y.) 1965.

[15] Ducuing, J., Proceedings of the International School of physics
« E. Fermi », Cours 42 (Edited by R. J. Glauber, Academic Press) 1969.

[16] Puthoff, H. E., Pantell, R. H., Huth, B. G., Chacon, M. A., J. Appl. Phys. 39 (1968) 2144.

[17] De Martini, F., Phys. Rev. B 4 (1971) 4556.

[18] Biraud-Laval, S., Chartier, G., Reinisch, R., Light Scattering in Solids (Flammarion, Paris) 1971.

[19] Zallen, R., Lucovsky, G., Taylor, W., Pinczuk, A., BursTEIN, E., Phys. Rev. B 1 (1970) 4058.

[20] Otaguro, W., Wiener-Avnear, E., Arguello, C. A., Porto, S. P. S., Phys. Rev. B 4 (1971) 4542.

[21] Rimai, L., Barsons, J. L., Hickmott, J. T., Nakamura, T., Phys. Rev. 168 (1968) 623.

[22] Alfano, R. R., J. Opt. Soc. Am. 60 (1970) 66.

[23] Alfano, R. R., Giallorenzi, T. G., Opt. Commun. 4 (1971) 271.

[24] Giallorenzi, T. G., Phys. Rev. B 5 (1972) 2314.

[25] Faust, W. L., Henry, C. H., Phys. Rev. Lett. 17 (1966) 1265.

[26] Miller, R. C., Appl. Phys. Lett. 5 (1964) 17.

[27] Shen, Y. R., Bloembergen, N., Phys. Rev. A 137 (1965) 1787.

[28] ReINISCH, R., Thèse d'Etat, Orsay (1975).

[29] Biraud-laval, S., Reinisch, R., Paraire, N., Laval, R., Phys. Rev. B 13 (1976). 